\title{
Epileptogenic Source Imaging Using Cross Frequency Coupled Signals from Scalp EEG
}

\author{
Chunsheng Li, Daniel Jacobs, Trevor Hilton, Martin del Campo, \\ Yotin Chinvarun, Peter L Carlen, and Berj L Bardakjian
}

\author{
Version Post-print/accepted manuscript \\ Citation C. Li et al., "Epileptogenic Source Imaging Using Cross-Frequency \\ (published version) Coupled Signals From Scalp EEG," in IEEE Transactions on \\ Biomedical Engineering, vol. 63, no. 12, pp. 2607-2618, Dec. 2016. \\ doi: 10.1109/TBME.2016.2613936 \\ Publisher's Statement C 2016 IEEE. Personal use of this material is permitted. \\ Permission from IEEE must be obtained for all other uses, in any \\ current or future media, including reprinting/republishing this \\ material for advertising or promotional purposes, creating new \\ collective works, for resale or redistribution to servers or lists, or \\ reuse of any copyrighted component of this work in other works.
}

How to cite TSpace items

Always cite the published version, so the author(s) will receive recognition through services that track citation counts, e.g. Scopus. If you need to cite the page number of the author manuscript from TSpace because you cannot access the published version, then cite the TSpace version in addition to the published version using the permanent URI (handle) found on the record page.

This article was made openly accessible by $U$ of 'T Faculty.

Please tell us how this access benefits you. Your story matters. 


\title{
Epileptogenic Source Imaging Using Cross Frequency Coupled Signals from Scalp EEG
}

\author{
Chunsheng Li, Daniel Jacobs, Trevor Hilton, Martin del Campo, Yotin Chinvarun, Peter L Carlen, \\ and Berj L Bardakjian, Member, IEEE
}

\begin{abstract}
Objective: The epileptogenic zone (EZ) is a brain region containing the sources of seizure genesis. Removal of the EZ is associated with cessation of seizures after resective surgical procedures, as measured by Engel Class I score. This study describes a novel EEG source imaging (ESI) method which uses cross frequency coupled potential signals $\left(\mathbf{S}_{\mathrm{CFC}}\right)$ derived from scalp EEG. Methods: Scalp EEG were recorded from ten patients (20 seizures) suffering from epilepsy. The $S_{C F C}$ were constructed from the phase and amplitude of the lower and higher frequency rhythms at electrographic seizure onset. ESI was then performed using the $S_{C F C}$. Validation of the technique was facilitated by (i) forward and inverse computer modelling of known cortical sources, and (ii) the correspondence of the ESI with EZ in resected regions of patients. Results: For ten seizures sampled at or above $500 \mathrm{~Hz}$ from four patients, all estimated sources lay within the resected region, emphasizing the clinical importance of higher sampling rates. The $S_{C F C}$ demonstrated significant advantages over the "raw" scalp EEG, indicating its robust noise performance. Modelling investigations indicated that a signal-to-noise ratio above 0.2 was sufficient to achieve successful localization regarding EMG artifacts. Conclusion: The association of the estimated sources to the EZ suggests that crossfrequency coupling is a feature of the brain's neural networks, not of artifactual activity. The $S_{C F C}$ can effectively extract brain signals from a noisy background. Significance: We propose this approach to enhance the placement of intracranial electrode for surgical intervention.
\end{abstract}

Index Terms-Epilepsy; EEG source imaging (ESI); cross frequency coupling; modulation index (MI); artifactual immunity.

\section{INTRODUCTION}

$\mathbf{E}$ PILEPSY is the most common chronic disease in neurology. More than $30 \%$ of epileptic patients are refractory to pharmacotherapy and may elect to undergo surgical

This work was supported by Natural Sciences and Engineering Research Council of Canada (NSERC) and Canadian Institutes of Health Research (CIHR). C. Li was also supported by the China Scholarship Council.

$\mathrm{C}$. Li is with the Institute of Biomaterials and Biomedical Engineering, University of Toronto, Toronto, M5S 3G9 Canada. He is also with School of Electrical Engineering, Shenyang University of Technology, Shenyang, 110870 China (e-mail: chunsheng.li@utornto.ca).

D. Jacobs and T. Hilton are with the Institute of Biomaterials and Biomedical Engineering, University of Toronto, Toronto, M5S 3G9 Canada.

M. del Campo is with the Department of Medicine (Neurology), Toronto Western Hospital, Toronto, M5T 2S8 Canada.

Y. Chinvarun is with the Comprehensive Epilepsy Program and Neurology Unit, Phramongkutklao Hospital, Bangkok, 10400 Thailand.

P. L. Carlen is with the Institute of Biomaterials and Biomedical Engineering, University of Toronto. He is also with Department of Medicine (Neurology), Toronto Western Hospital and Krembil Research Institute, University Health Network, Toronto, M5T 2S8 Canada.

B. L. Bardakjian is with the Institute of Biomaterials and Biomedical Engineering and the Department of Electrical and Computer Engineering, University of Toronto, Toronto, M5S 3G4 Canada (e-mail:berj@cbl.utoronto.ca). intervention [1]. The goal is to remove the epileptogenic zone (EZ), which is the brain tissue necessary for seizure generation [2], [3]. Clinicians perform pre-surgical evaluation using both noninvasive and invasive tools to estimate the EZ. Scalp electroencephalography (EEG) is one of these techniques which is fundamental for defining the EZ, frequently as a precursor to invasive recordings in the form of intracranial EEG (iEEG). iEEG is the gold standard for EZ definition, but has certain limitations, chiefly the potential for incomplete detection of the EZ because of limited spatial coverage [3], [4]. As such, correct placement of iEEG electrodes is crucial for surgical success, but it remains a challenge due to the effects of volume conduction of brain rhythms from their source to the scalp, where they may be further obscured due to the presence of strong artifacts, such as from muscle activity. Such difficulties may contribute to the $30 \%$ of surgical resections which fail to produce seizure freedom. As such, improved methods for noninvasive mapping of epileptogenic networks across the whole brain are critical for the appropriate presurgical evaluation [5].

EEG source imaging (ESI) is a technique that has been used extensively for noninvasive mapping of the brain's neural activity [6]-[8] and has particular clinical value in its ability to estimate epileptic foci [9]-[11]. ESI allows for the enhancement of the analysis of potential fields recorded on the scalp by first inverting them to the cortical source space and conducting analyses such as connectivity and coherence in the cortical source domain [12], [13]. ESI techniques have also been enhanced by processing the potential scalp field before inversion, for example: a) transforming the scalp potential field to the frequency domain using the Fourier transform [14], b) generalization of the FFT approximation to the case of distributed source models with non-stationary time behavior [15], and c) time-frequency MEG-MUSIC algorithm [16]. Most ESI studies for pre-surgical epilepsy evaluation have relied on interictal changes rather than ictal activity [17]. There have been few studies dealing with ictal source localization [18], [19]. This may be due to the difficulty of ictal source modeling: the low signal to noise ratio of some ictal pattern types, and their frequent contamination by artifacts [9]. Artifacts may be introduced by eye movement, eye blinks, electrode movement, muscle activity, breathing, heartbeat, and electrical line noise [20]. However, ictal EEG patterns are essential for determining the origin and spread of epileptic activity [21]. Isolation of EZspecific activity in the presence of these artifacts in the ictal EEG could lead to improved ESI for mapping epileptogenic networks. 
Interaction between neuronal rhythms may offer a novel method of selecting EZ-specific information for ESI techniques. Rhythmic interaction was found to have a fundamental and significant role in high level brain function such as cognition and memory [22]-[24]. In epileptic patients, elevated low frequency oscillation and high frequency oscillation coherence identified coinciding regions of interest (ROIs) in the intracranial recordings [25]. High frequency amplitude modulated by low frequency phase has been used to successfully define ROI on intracranial recording [26]. Low and high frequency oscillation have long been studied independently in the context of epilepsy. Delta asymmetry has been found to be a useful marker for lateralizing the epileptogenic focus [27]. The presence of delta slow waves was prevalent in patients with uncontrolled seizures [28]. Specially, both temporal and occipital intermittent rhythmic delta activities were highly correlated with epilepsy [29]. Interictal regional delta slowing has also been found to correlate with positive surgical outcomes in patients with temporal lobe epilepsy [30], [31]. With regards to high frequency oscillation, the presence of ripples was shown to be significant in the seizure onset zone (SOZ) of patients with neocortical epilepsy [32], and the resection of high frequency oscillation generating tissue has been linked to positive surgical outcome in both adults [33]-[35] and children [36], [37]. There has been a renewed interest for the study of fast oscillations with scalp EEG [38]. So far, only a few studies using scalp EEG in humans have demonstrated cross-frequency coupling between low frequency phase and high frequency amplitude in the context of working memory and attention tasks [39], [40]), and no studies have yet investigated cross frequency coupling in scalp EEG recordings from epileptic patient.

The present study proposes a novel EZ source imaging method using cross frequency coupled potential signals $\left(\mathrm{S}_{\mathrm{CFC}}\right)$ formed by the phase feature of the lower frequency range (LFR) and the amplitude feature of the higher frequency range (HFR). This method is validated via (i) simulation, and (ii) comparison between $\mathrm{S}_{\mathrm{CFC}}$-localized sources of the ROI (sROI) and the known resected regions, the $\mathrm{S}_{\mathrm{CFC}}$ signal demonstrates advantages over the original scalp EEG. For patients with Engel Class I outcome the sROI was checked to be in the resected region, otherwise some or all sources were localized outside that region.

\section{MATERIALS AND METHODS}

\section{A. Data and subject description}

Twenty seizures were obtained in the form of scalp EEG recordings from ten patients with intractable epilepsy. Informed consent was obtained from each patient and the ethics committees of the affiliated institutions approved this study. Six patients were seizure free after surgical intervention. The clinical background as well as surgical outcome of each patient is outlined in Table I. Note that for patient P1, there was a resection performed, but the surgical outcome is not known because this patient died of unknown causes within the first few weeks after surgery. In this patient's case, the EZ was assumed to be in accordance with the resection made. Global

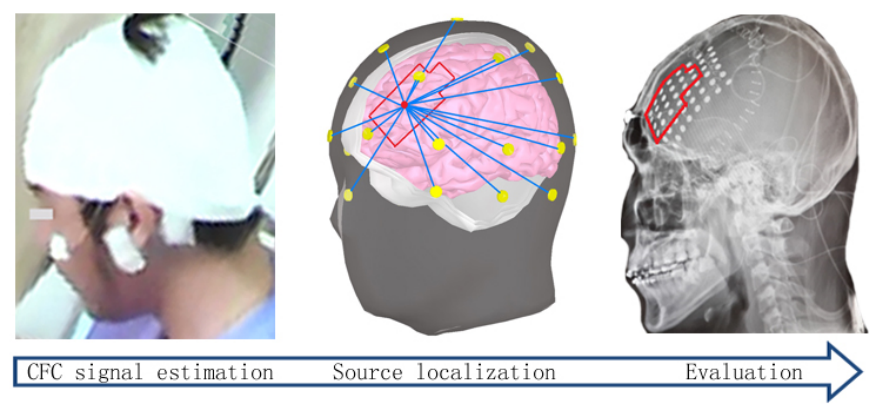

Fig. 1. Scalp EEG, head model, and surgical resection. Inverse modelling using the $\mathrm{S}_{\mathrm{CFC}}$ signal derived from the scalp EEG produced cortical source estimates, which could be evaluated relative to the known surgical resection.

reference electrode $(\mathrm{Fpz})$ was used in recording for patients P1-P3, P5, P7, and post-auricular locations were used for patient P4, P6, P8-P10. The number of scalp electrodes used for source localization varied between patients from 19 to 25 , and recordings were acquired with various sampling frequencies from 200 to $1000 \mathrm{~Hz}$ (summarized in Table II). Line noise interference was notched with a zero-phase Butterworth filter. Two neurologists examined the recordings and identified electrographic SOZs. Patient specific CT imaging was used to confirm intracranial electrode positions. The electrographic onset of the seizure was essential for determining SOZ. EEG segments of $10 \mathrm{~s}$ duration $\left(t_{\mathrm{sp}}\right)$ starting from electrographic seizure onset, as determined by the neurologists, were chosen for all subsequent analyses. Recordings of $10 \mathrm{~s}$ duration appear sufficient to determine ROIs in epileptic patients [26]. Patient P1 and P3 had seizures with intracranial and extracranial EEG simultaneously recorded. Scalp EEG at electrographic seizure onset was used for deriving the $\mathrm{S}_{\mathrm{CFC}}$ signal, and the source localization result was compared with resected region of patient for validation (see Fig. 1).

\section{B. Cross frequency coupled potential signals}

$S_{\mathrm{CFC}}(t)$ is proposed as a time varying potential signal, which is derived from a HFR amplitude signal, $A_{\mathrm{HFR}}(t)$, and a LFR phase signal, $\phi_{\mathrm{LFR}}(t)$, of scalp EEG. The $\mathrm{S}_{\mathrm{CFC}}$ signal at each electrode is defined as follows:

$$
S_{\mathrm{CFC}}(t)=A_{\mathrm{HFR}}(t) \cdot \sin \left[\phi_{\mathrm{LFR}}(t)\right],
$$

where

$$
\begin{aligned}
A_{\mathrm{HFR}}(t) & =\left.\langle A(t, f)\rangle\right|_{f \in\left[H_{1}, H_{2}\right]}, \\
\phi_{\mathrm{LFR}}(t) & =\left.\langle\phi(t, f)\rangle\right|_{f \in\left[L_{1}, L_{2}\right]},
\end{aligned}
$$

and $\langle\cdot\rangle$ denotes average value.

Continuous wavelet transform (CWT) was applied on each electrode in an EEG recording (e.g. Fig. 2(c)), $x(t)$, in order to generate the time-frequency spectrum. This produced a complex coefficient at each point in time $t$ and frequency $f$, whose amplitude and phase were the $A(t, f)$ and $\phi(t, f)$ respectively. All computations were performed within the MATLAB environment (The MathWorks, Natick, MA, U.S.A.). Assuming $A_{\mathrm{HFR}}(t) \cdot e^{j \phi_{\mathrm{LFR}}(t)}$ is analytic, then the $\mathrm{S}_{\mathrm{CFC}}$ signal would be 
TABLE I

CLINICAL BACKGROUND OF PATIENT DATASET

\begin{tabular}{|c|c|c|c|c|c|c|c|c|}
\hline P\# & Age/Sex & $\begin{array}{c}\text { Duration } \\
\text { (years) }\end{array}$ & $\begin{array}{l}\text { Number of } \\
\text { Channels }\end{array}$ & $\begin{array}{l}\text { Intracranial } \\
\text { Electrode }\end{array}$ & $\begin{array}{l}\text { MRI } \\
\text { Findings }\end{array}$ & Pathology & Resection & $\begin{array}{l}\text { Surgical } \\
\text { Outcome }\end{array}$ \\
\hline $\mathrm{P} 1 *$ & $30 \mathrm{M}$ & 26 & 25 & Strips & $\begin{array}{l}\text { Small left inferior } \\
\text { frontal encephalocele }\end{array}$ & $\begin{array}{l}\text { Minimal white matter } \\
\text { vascular changes }\end{array}$ & Left: FT & - \\
\hline $\mathrm{P} 2$ & $41 \mathrm{M}$ & 14 & 25 & Strips, Deep & Mesial temporal sclerosis & Mesial temporal sclerosis & Right: T & EC I \\
\hline $\mathrm{P} 3 *$ & $26 \mathrm{~F}$ & 4 & 25 & Strips, Deep & Normal & Normal & Right: F & EC I \\
\hline P4 & $26 \mathrm{~F}$ & 9 & 19 & Grid & $\begin{array}{l}\text { Thickening cortex of } \\
\text { anterior cingulate gyrus }\end{array}$ & Neuronal heterotropia & Left: F & EC I \\
\hline P5 & $22 \mathrm{M}$ & 9 & 25 & Strips, Deep & Normal & Normal & Left: $\mathrm{T}$ & EC I \\
\hline P6 & $21 \mathrm{M}$ & - & 19 & Grid & Cortical dysplasia & Normal & Left: DF & EC I \\
\hline $\mathrm{P} 7$ & $31 \mathrm{M}$ & 22 & 24 & Strips, Deep & Mesial temporal sclerosis & Mesial temporal sclerosis & Right: $\mathrm{T}$ & EC I \\
\hline P8 & $56 \mathrm{~F}$ & 8 & 19 & Grid & Nonlesional & Cortical microdysgenesis & Left: $\mathrm{P}$ & EC II \\
\hline P9 & $28 \mathrm{M}$ & 16 & 19 & Grid & Nonlesional & Cortical microdysgenesis & Left: FT & EC III \\
\hline P10 & $16 \mathrm{M}$ & 4 & 19 & Grid & Cortical dysplasia & Normal & Right: DF & EC IV \\
\hline
\end{tabular}

F: frontal; T: temporal; D: dorsolateral; P: parietal; O: occipital; *: simultaneous recording; -: unknown; P\#: patient number; EC: Engel Class

(Engel 1993).

the Hilbert transform of the signal $A_{\mathrm{HFR}}(t) \cdot \cos \left[\phi_{\mathrm{LFR}}(t)\right]$ (see Fig. 2(d)). The $\mathrm{S}_{\mathrm{CFC}}$ signal from seizure one of patient $\mathrm{P} 3$ is shown in Fig. 2(e). The estimation of LFR $\in\left[L_{1}, L_{2}\right]$ and HFR $\in\left[H_{1}, H_{2}\right]$ will be illustrated in following subsection.

\section{Higher and lower frequency ranges from global modulation index}

The HFR and LFR were estimated from the global modulation index (GMI), which is the average of modulation index (MI) of all channels [41], [42]. To compute the MI, the time series of the amplitude envelope of higher frequency signal and the instantaneous phase of lower frequency signal were extracted from their respective CWT spectra, which were obtained by using the complex Morlet wavelet with a bandwidth of $5 \mathrm{~Hz}$ and a center frequency of $0.8125 \mathrm{~Hz}$ [26]. The low frequency phase was divided into 18 bins, and high frequency amplitude within each phase bin was averaged. The mean amplitude was then normalized by dividing the sum of all mean amplitudes. The normalized amplitude had discrete probability density function characteristics, and was then compared with a uniform distribution by measuring the Kullback-Leibler (KL) distance. The KL distance was normalized to values between 0 and 1, defining the MI [41].

The HFR and LFR were selected by taking the maximal extent of the GMI distribution at or above $3 \mathrm{~dB}$ below peak. For the computation of each individual MI map, the higher frequency range was chosen as being greater than $10 \mathrm{~Hz}$ in 1 $\mathrm{Hz}$ increments, whereby the lower frequency range was chosen as being less than or equal to $8 \mathrm{~Hz}$ in $0.1 \mathrm{~Hz}$ increments. The MI values at electrographic onset were computed. Scalp EEG at electrographic onset of P3 was showed in Fig. 2(a). The GMI was shown in Fig. 2(b), which highlighted the interaction region at $\mathrm{LFR} \in[3.0,4.2] \mathrm{Hz}$ and $\mathrm{HFR} \in[20,50] \mathrm{Hz}$. The CWT spectrum in LFR and HFR at channel F4 were shown in Fig. 2(c), and those regions were further converted to $\mathrm{S}_{\mathrm{CFC}}$ time varying potential signal at corresponding channel.

To determine whether the GMI is highlighting electrical activity in the scalp EEG recordings, the simultaneous EEG and iEEG recordings were utilized from patient P3, seizure one, during the $t_{\mathrm{sp}}$. Cortical current sources were approximated at the locations of each iEEG recording electrode (see Fig. 7(d)) so that, using the forward conduction model, resulting potentials at standard scalp EEG locations could be generated. This gave two signals for each standard scalp EEG location: a simulated signal representing the neuronal component of the EEG as derived from the simultaneous iEEG recordings, and the original recorded EEG signal. To select reliable sites for comparison of the two signals, unit dipole sources were placed at all iEEG source locations in the model. The resulting scalp potential distribution is observed in Fig. 3(a). By selecting a threshold of $3 \mathrm{~dB}$ below the maximum power in this distribution, two channel locations: F4 and F8, the only channels whose power was above this threshold, were chosen. At these locations, the two simultaneous signals were compared on a per-frequency basis using two separate methods: Wavelet phase coherence (WPC) [43] and Wavelet envelope correlation (WEC) [44]. In Fig. 3(b), it is seen that there exists an elevation in WPC within the frequency range selected by the GMI at both channel locations. In Fig. 3(c), a similar elevation is seen in the WEC for the frequency range selected by the GMI. Averaged MI from projected iEEG and recorded EEG at channel F4 and F8 (see Fig. 3(d)) showed same HFR and LFR ranges as GMI (see Fig. 2(b)). This indicates that the GMI is able to select frequency components of the scalp EEG that are the result of neuronal activity and not 


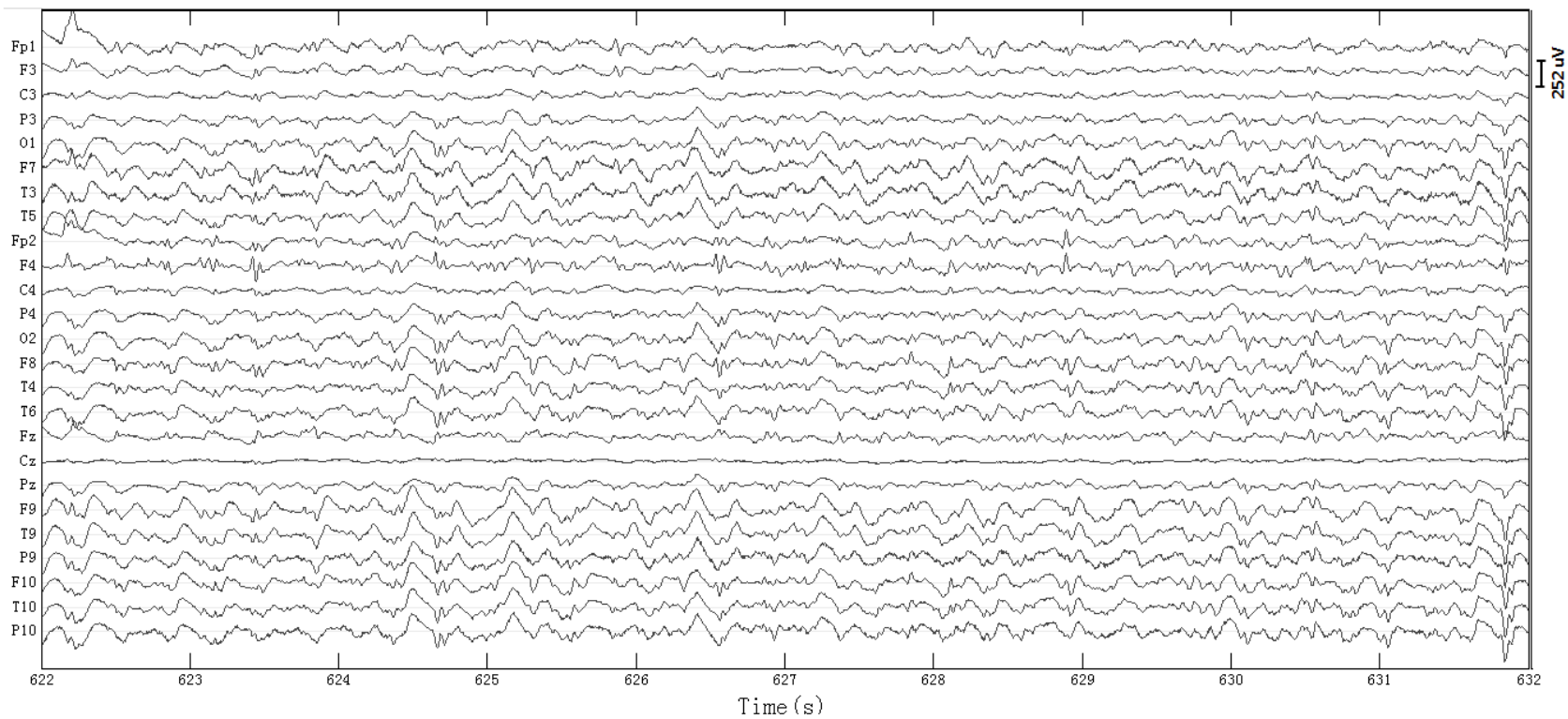

(a)

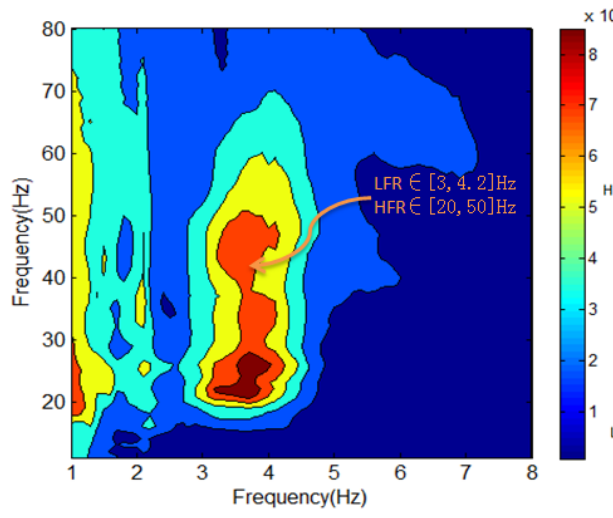

(b)

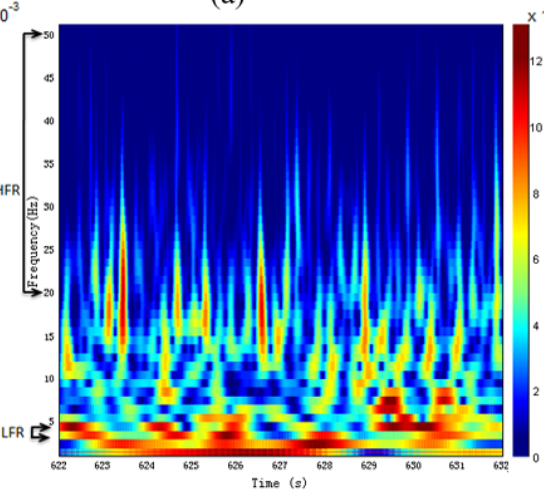

(c)

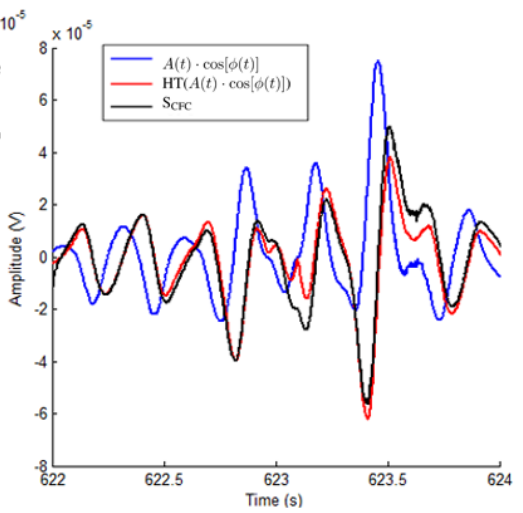

(d)

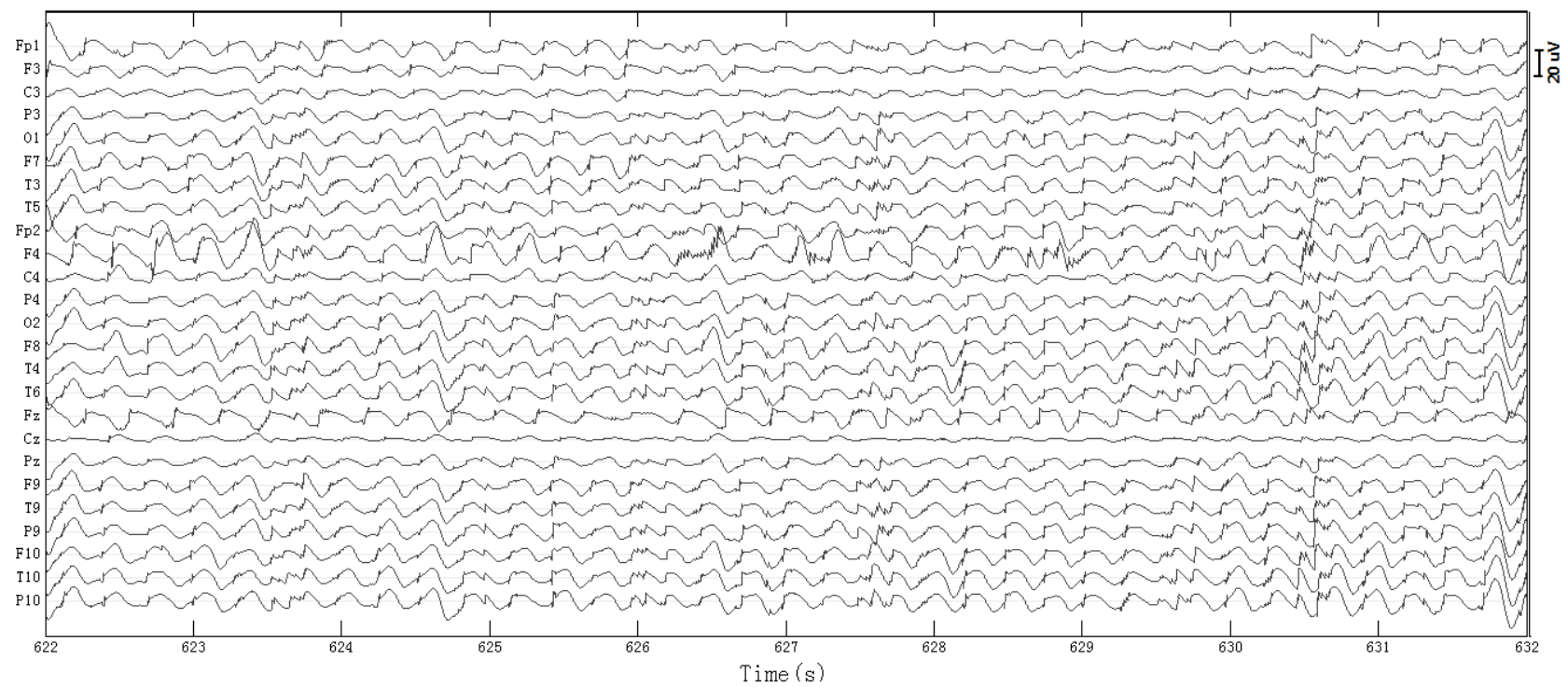

(e)

Fig. 2. The $\mathrm{S}_{\mathrm{CFC}}$ signal derived from raw scalp EEG. (a) Raw 25 channels $10 \mathrm{~s}$ scalp EEG at electrographic seizure onset from patient $\mathrm{P} 3$ seizure one. (b) GMI averaged from 25 channels MI. Low frequency range $1-8 \mathrm{~Hz}$ and high frequency range $11-80 \mathrm{~Hz}$ were used. The region marked by a white arrow indicates the coupled LFR and HFR. LFR $\in[3,4.2] \mathrm{Hz}, \mathrm{HFR} \in[20,50] \mathrm{Hz}$. (c) CWT at channel F4. HFR and LFR were marked, and $\mathrm{S}_{\mathrm{CFC}}$ signal was derived from averaged traces from those ranges. (d) The $\mathrm{S}_{\mathrm{CFC}}$ signal computed from patient P3's channel $\mathrm{F} 4$ with lower and higher frequencies selected at $3.7 \mathrm{~Hz}$ and $26 \mathrm{~Hz}$. The $\mathrm{S}_{\mathrm{CFC}}$ signal is the Hilbert transform (HT) of the signal $A(t) \cdot \cos [\phi(t)]$. (e) The $\mathrm{S}_{\mathrm{CFC}}$ signal derived from the selected LFR and HFR range of (b). 


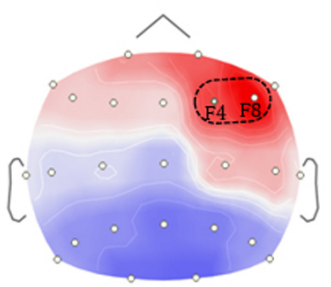

(a)

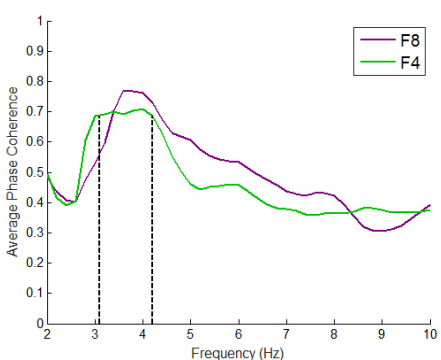

(b)

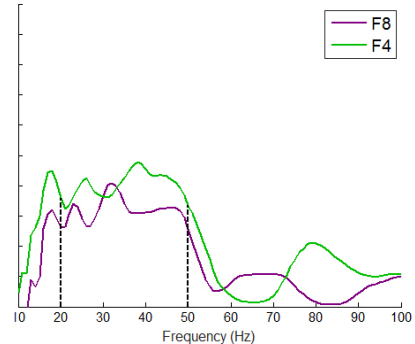

(c)

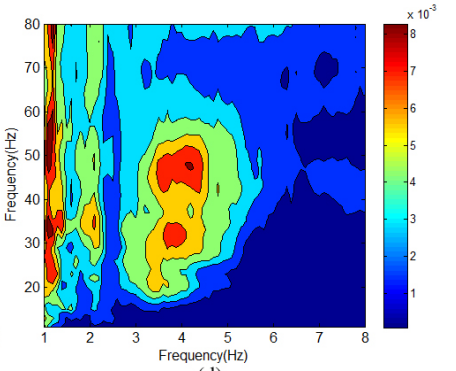

(d)

Fig. 3. Validation of $\mathrm{S}_{\mathrm{CFC}}$ signal LFR and HFR selection. (a) Two channels: F4 and F8 were selected (dashed circle) for their power being above the 3 $\mathrm{dB}$ below the maximum power in unit source forward projected distribution. (b) Average WPC between the iEEG projected and recorded EEG signals at channel F4 and P8 of patient P3's seizure one, which was shown in Fig. 2(a). LFR showed plateau with high phase coherence. (c) Average WEC between the iEEG projected and recorded EEG signals at channel F4 and F8 of patient P3's seizure one. HFR range showed plateau with high amplitude coherence. (d) Averaged MI from projected iEEG and recorded EEG at channel F4 and F8.

those that may be of artifactual origin. Since the LFR and HFR of $\mathrm{S}_{\mathrm{CFC}}$ signal were chosen from maximally coupled neuronal rhythms, this phase selectivity can eliminate artifactual effects as seen in Fig. 4.

\section{Source localization}

ESI was performed using the Brainstorm software package [45] within MATLAB. weighted minimum norm estimate (wMNE) was the primary method for ESI [46]. Standard lowresolution brain electromagnetic tomography (sLORETA) was also used for comparative purposes [47]. The MNI Colin 27 brain was used for both forward and inverse modeling [48], see Fig. 1. A four-layer (skull, scalp, cerebral spinal fluid, and cortex) boundary element method model was obtained through the OpenMEEG software package [49]. With this model, neuronal source activity was estimated using the aforementioned ESI techniques. Average source strength was computed over the $t_{\mathrm{sp}}$, and significant activity was defined as that above the threshold of $3 \mathrm{~dB}$ below (i.e. $70 \%$ of) the maximum average source strength within this interval.

\section{RESULTS}

\section{A. Validation via simulation}

The comparison between $\mathrm{S}_{\mathrm{CFC}}$ mapping applied to raw scalp EEG and their cortical sources was investigated. Raw scalp EEG sources from seizure one of patient P3 are displayed in Fig. 5(a). The raw EEG sources were localized on the left temporal lobe while the brain resection of this patient was on the right frontal lobe. The $\mathrm{S}_{\mathrm{CFC}}$ signals were estimated for all cortical source signals, with the HFR and LFR selected from the raw scalp EEG GMI-based ranges (in Fig. 2(b)). Those source strengths were averaged over the $t_{\mathrm{sp}}$, normalized according to maximum value. Most of the $S_{\mathrm{CFC}}$ signal activities above $3 \mathrm{~dB}$-below-max threshold were localized in the right frontal lobe (see Fig. 5(b)). The $\mathrm{S}_{\mathrm{CFC}}$ mapping in sensor space applied directly to scalp EEG were localized in the right frontal lobe as seen in Fig. 5(c).

Instead of using the raw scalp EEG for ESI, the scalp EEG were finite impulse response (FIR)-filtered into the LFR and HFR. The HFR and LFR sources were both localized over the frontal and left temporal lobes as seen in Fig. 6(a) and (b).
The $\mathrm{S}_{\mathrm{CFC}}$ signals were estimated in the source space using the combined LFR and HFR sources. The resulting potential map was time-averaged to estimate the location of the EZ, which was predominantly localized in the right frontal lobe as seen in Fig. 6(c). However, this result was not as good as $\mathrm{S}_{\mathrm{CFC}}$ mapping in sensor space in Fig. 5(c).

In order to validate the ability of the $S_{\mathrm{CFC}}$ to estimate the EZ under electromyographic (EMG) contamination, simultaneous scalp and iEEG recordings from seizure one of patient P3 (Engel class I) were used. The iEEG recording appears to be minimally affected by EMG and other artifacts, and is assumed to represent activity of mostly cortical origin [50], [51]. iEEG was projected to the scalp electrode locations using the forward model. The amplitude of the projected traces was scaled according to variance matching by the ratio of the standard deviation of the projection to that of the scalp EEG recording at the F4 electrode. This scaling was performed in order to match the amplitude of the projected signal to the scalp recordings. F4 was selected since the projection at that location matches well with the scalp recording (see Fig. 3), and also has the largest standard deviation. This set of projected signals (see Fig. 7(a)) was treated as an artifact-free subset of the recorded scalp signal. The $\mathrm{S}_{\mathrm{CFC}}$ source estimate for this noise-free case defines an ROI used to assess the effects of added EMG noise. This ROI coincides with the known resection area (see Fig. 7(d) and (e)).

Realistic EMG was introduced into this noise-free model using the simultaneously recorded scalp EEG. EMG-type components were separated from EEG activity (see Fig. 7(b)) via independent component analysis (Infomax ICA) [52]. EMG could then be scaled to the desired signal-to-noise ratio (SNR). SNR scaling was computed using the frequency range from the spectrum of the traces with the largest SNR, as reported in other simulation work [53]. Frequencies were first binned into ranges of interest following the frequency ranges seen across patients while computing the $\mathrm{S}_{\mathrm{CFC}}$ (see Table II).

An example of deliberately contaminated signal is shown in Fig. 7(c). EMG is added to electrode T5 at an SNR of 0.1 , and the EMG decays across the entire scalp as seen in previous studies of the spatial extent of EMG contamination of EEG [54]. To ensure widespread contamination, the EMG was made to decay linearly with distance (as opposed to expo- 


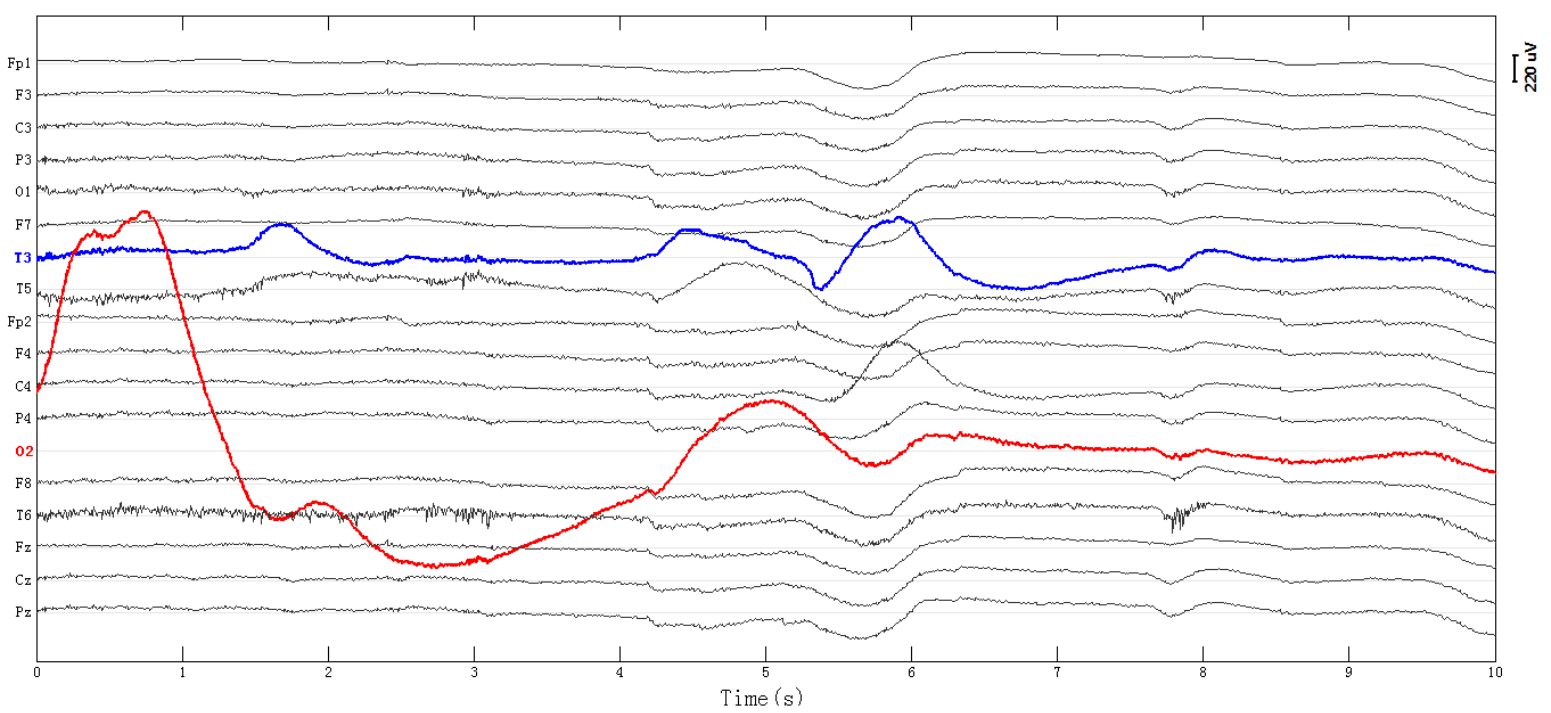

(a)

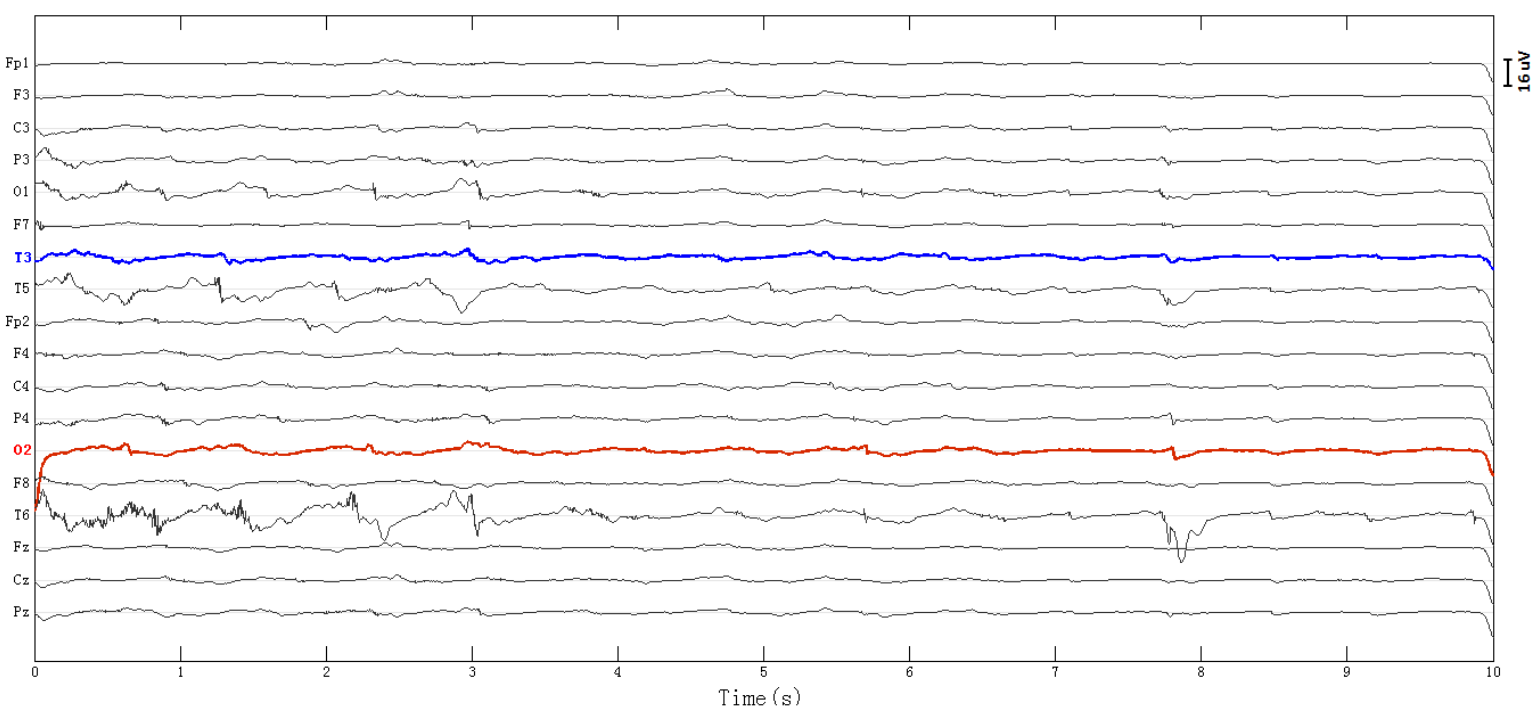

(b)

Fig. 4. Scalp EEG and derived $\mathrm{S}_{\mathrm{CFC}}$ signal before seizure termination of patient $\mathrm{P} 9$. (a) $10 \mathrm{~s}$ raw scalp EEG. A large artifact was shown on electrode O2 (red line) and T3 (blue line). All channels show baseline changing between 4 to $6 \mathrm{~s}$. (b) $\mathrm{S}_{\mathrm{CFC}}$ signal derived from corresponding scalp EEG. The artifacts on channel $\mathrm{O} 2$ and T3 were removed, and the abnormal baseline at 4 to $6 \mathrm{~s}$ was also removed. The LFR $\in[1.1,1.4] \mathrm{Hz}$ and $\mathrm{HFR} \in[41,51] \mathrm{Hz}$ were determined from corresponding GMI.

nentially) from $\mathrm{T} 5$. $\mathrm{S}_{\mathrm{CFC}}$ signals were then constructed from each contaminated set and used for imaging. The proposed $\mathrm{S}_{\mathrm{CFC}}$ method demonstrates the ability to localize the ROI even under the influence of strong noise, as shown for the example in Fig. 7(f) which matches the noise-free case. A similar, although more diffuse, localization result is achieved using sLORETA instead of the wMNE method (see Fig. 7(g)).

The effects of EMG artifacts and white noise upon the sensitivity and specificity of the $\mathrm{S}_{\mathrm{CFC}}$ technique were then evaluated across individual channels, for a range of SNR values. Particularly low SNR recordings (such as that in Fig. 8(a) and (c)) may demonstrate activity outside the ROI. To define a successful ESI result, we define $S_{\text {in }}$ as the percentage of the ROI recovered by a given source estimate (sensitivity), and $S_{\text {out }}$ as the percentage of non-ROI identified in a given estimate (specificity). For this specific seizure event, the imaging was generally successful as per these measures across channels for SNR greater than 0.2 in EMG artifacts and 0.75 in Gaussian white noise (see Fig. 8(e) and (f)). (This SNR threshold is met in the scalp EEG of this patient; in frequencies above $30 \mathrm{~Hz}$ for the $2 \mathrm{~s}$ period during which EMG-type activity is strongest, the SNR in the scalp is estimated at 0.5.) The $\mathrm{S}_{\mathrm{CFC}}$ technique produces more stable imaging when noise is introduced further away from the known EZ. For 'edge-case' SNR trials, the desired sROI could be reliably identified by increasing the threshold of the source estimate $\left(S_{\mathrm{th}}\right)$ beyond the 3 dB-below-max (see Fig. 8(b) and (d)). 

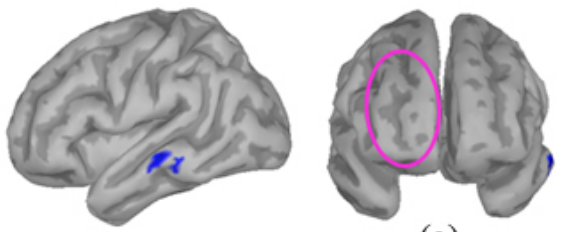

(a)
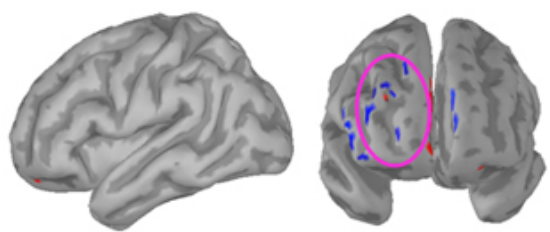

(b)
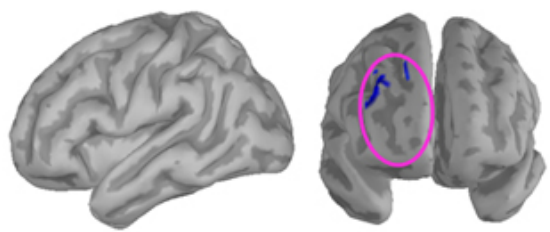

(c)

Fig. 5. ESI from $\mathrm{S}_{\mathrm{CFC}}$ and $\mathrm{EEG}$ potential maps for left, frontal, and right views. Purple boundary marks the brain resected area. All source activities were averaged over $10 \mathrm{~s}$, normalized according to maximum values, and 3 dB-below-max value was chosen as threshold. EEG segment from seizure one of patient P3 was used. (a) Sources localized from raw scalp EEG. (b) $\mathrm{S}_{\mathrm{CFC}}$ mapping of cortical sources from (a). (c) ESI from $\mathrm{S}_{\mathrm{CFC}}$ mapped $\mathrm{EEG}$.
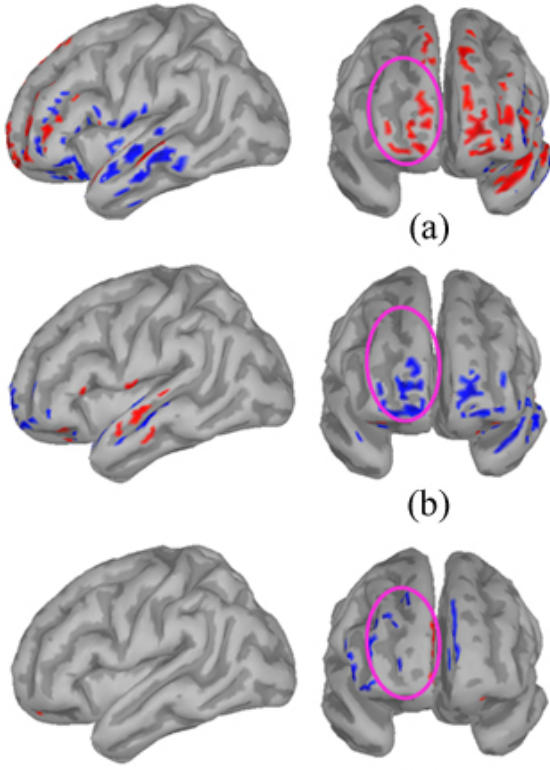

(c)

Fig. 6. ESI from (a) LFR and (b) HFR filtered EEG potential maps for left, frontal, and right views. Purple boundary marks the brain resected area. All source activities were averaged over $10 \mathrm{~s}$, normalized according to maximum values, and $3 \mathrm{~dB}$-below-max value was chosen as threshold. EEG segment from seizure one of patient $\mathrm{P} 3$ was used. (c) ESI from $\mathrm{S}_{\mathrm{CFC}}$ mapping of cortical sources from (a) and (b).

\section{B. Comparison of sROI with surgical resection}

1) The results of $S_{\mathrm{CFC}}$ and "raw" localizations are summarized for all patients and seizures in Table II. Eighty-two percent (14/17) of seizures from Engel Class I patients were concordant with resected regions. Examples of this successful localization are shown for multiple patients in Fig. 5(c) and Fig. 9. The sROI from seizure one of patient P3 is displayed in Fig. 5(c). Patient P3 had right-frontal cortical epilepsy. The resected region of patient $\mathrm{P} 3$ was marked by purple lines, and the sROI was inside the known resected region. Therefore, the sROI properly identified the EZ since this patient became seizure free. The sROI and resected region of patient P2 were plotted in Fig. 9(b). Patient P2 suffered from right temporal epilepsy, and in each seizure the sROI was concordant with the resected region.

2) Patients with Engel Class I score: There were three patients (P2, P4, and P6) with three seizures and three patients (P3, P5, and P7) with two seizures in the dataset. All three seizures of patient $\mathrm{P} 2$ and $\mathrm{P} 4$ achieved positive localization. For patient P6, sROI from seizure one was concordant with resected region, but sROI from seizure two and three were shown on the left and the right temporal lobe. For patient P3 and P5, the two sROI were concordant with the resected region. For patient P7, sROI from seizure one was concordant with resected region. This patient's second seizure yielded stronger activity on the left temporal rather than the right temporal lobe. Four Engel Class I patients (P2-P5) showed consistent results between seizures.

3) Patients with scores greater than Engel Class I: Patient P8, P9, and P10 were classified into Engel Class II, III, and IV respectively. Accordingly, we would expect to find sources outside of the resected regions. Both patient P8 and P9 were diagnosed with cortical microdysgenesis, and patient P10 was diagnosed with cortical dysplasia based on MRI. The sROI of patient P8 is shown in Fig. 9(a). The resected region of patient P8 was at the left parietal lobe. The sROI was close to the resected region, but there was also another region shown at the left occipital lobe. In this case, the result indicated patient P8 may have two active regions at seizure onset. Comparing the sROI with the resected region shows that part of the sROI was outside the grid coverage. The sROI of patient P9 is shown in Fig. 9(a). The sROI was at the left occipital lobe, which was out of grid coverage. The sROI of patient P10 is also shown in Fig. 9(a). The sROI was at the right frontal and temporal lobes. The resected region of patient $\mathrm{P} 10$ was at right front lobe, close to the front localized region. However, the temporal lobe was not covered by grid in this patient, so there was no possibility to identify that region by intracranial recording.

4) The localized region of patient P3's seizure one using raw scalp EEG was plotted in Fig. 5(a), but the localized region was present on the wrong hemisphere, whereas the sROI was concordant with resected region. The raw EEG localized results for all patients are shown in column Raw of Table II, and none of them was consistent with resected region in Engel class I patients. Localization of the $\mathrm{S}_{\mathrm{CFC}}$ showed improvement compared with the original signal. Fig. 4(a) showed a 10 $\mathrm{s}$ scalp EEG before seizure termination of patient $\mathrm{P} 9$, and corresponding $\mathrm{S}_{\mathrm{CFC}}$ signal was shown in Fig. 4(b). There were large artifacts on channel $\mathrm{O} 2$ and $\mathrm{T} 3$ during seizure of patient $\mathrm{P} 9$, and there were also obviously artifacts on each channel at 4-6 s. The corresponding $\mathrm{S}_{\mathrm{CFC}}$ signal showed the artifacts of channel $\mathrm{O} 2$ and other channels at 4-6 s were removed. 


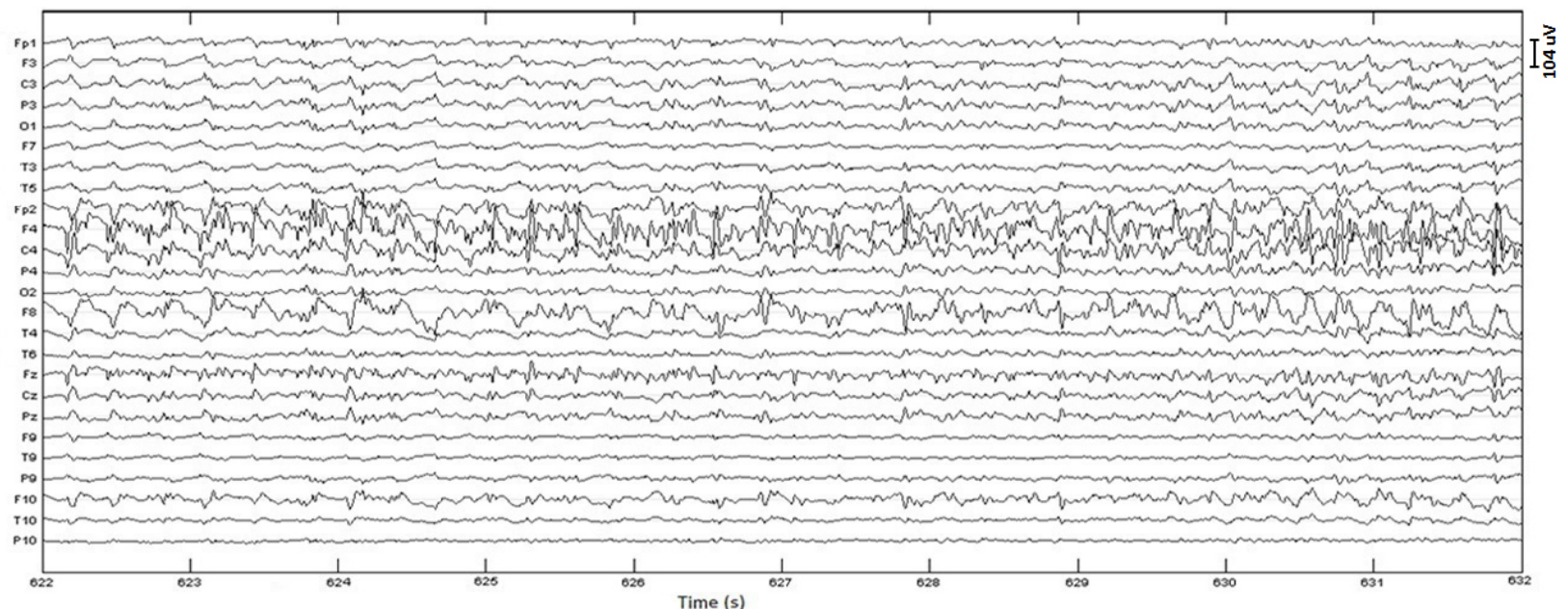

(a)

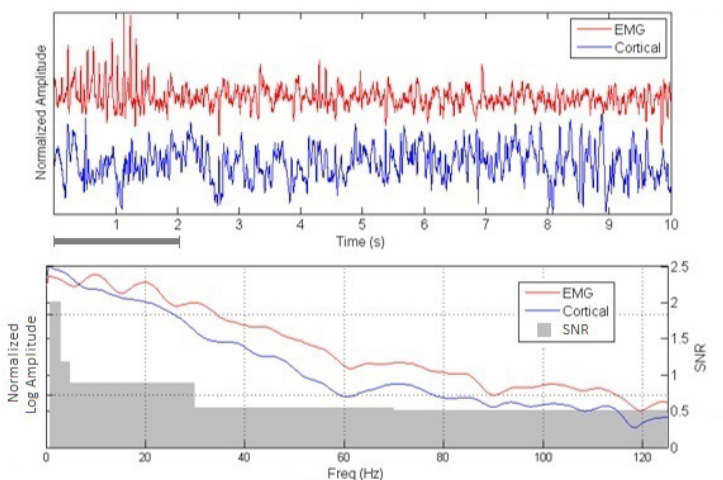

(b)

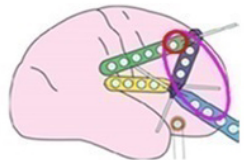

(d)

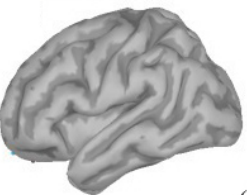

(e)

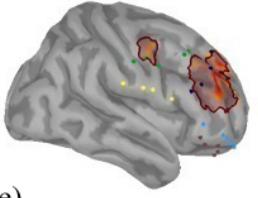

(e)

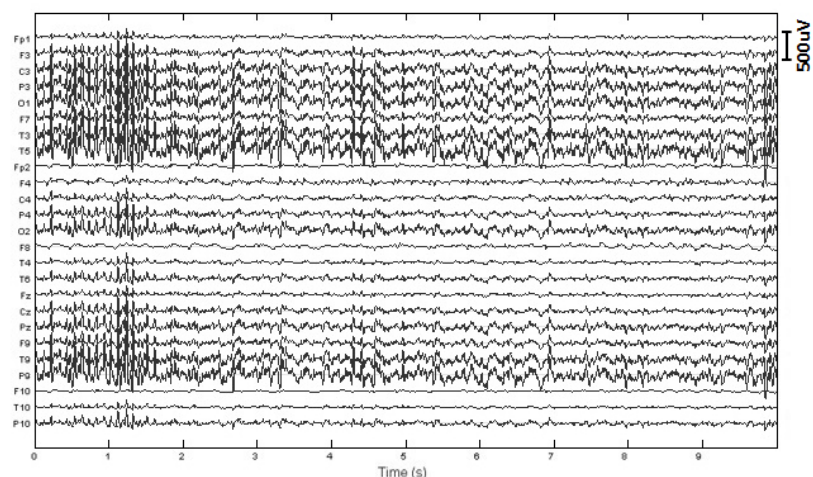

(c)

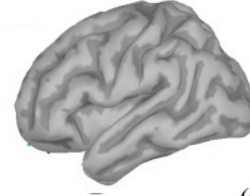

(f)
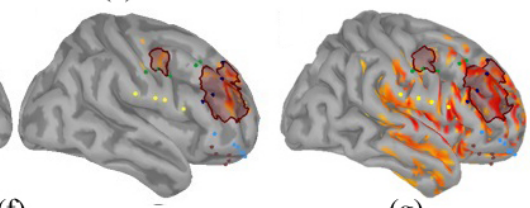

(g)

Fig. 7. $\mathrm{S}_{\mathrm{CFC}}$ source localization remains accurate in the presence of EMG artifacts. (a) Projection of intracranial recordings to scalp electrode positions of patient P3. The amplitude of the projected traces was scaled according to variance matching by the ratio of the standard deviation of the projection to that of the scalp EEG recording at the F4 electrode. (b) EMG artifact components from scalp EEG, shown at F9. The SNR is estimated by the ratio of the power spectrum post-binning into frequency ranges of interest $(1-3 \mathrm{~Hz}, 3-5 \mathrm{~Hz}, 5-30 \mathrm{~Hz}, 30-70 \mathrm{~Hz}$, and $70-125 \mathrm{~Hz})$. An SNR $>0.5$ is estimated for the initial 2 s. (c) EMG contamination centered at T5, SNR of 0.1. (d) Intracranial electrode positions for patient P3. Purple ellipse marks the resected region; red circle marks electrode RPF6 showing seizure onset. (e) Artifact-free sROI, which corresponds with resected region for the patient. (f) wMNE-produced sROI for contaminated signals (c) matches artifact-free sROI. (g) sLORETA-produced sROI for contaminated signals is comparable to wMNE-produced sROI.

\section{Discussion}

\section{A. $S_{C F C}$ signal properties}

The potential field of the scalp EEG was mapped into a sensor field of cross frequency coupled signals, which consist of two frequency ranges, a HFR and a LFR. Those ranges were estimated from GMI of scalp EEG potential field. The $\mathrm{S}_{\mathrm{CFC}}$ field is a mapped potential field since the $S_{C F C}$ signals have the unit of potential as defined in (1). By this approach, the scalp voltage distribution was mapped to emphasize pathological coupled activity [26]. While the $\mathrm{S}_{\mathrm{CFC}}$ has proven useful and effective, its physical interpretation is not clear as of yet, hence this stresses the need for ongoing research into its meaning. Our results demonstrate that sensor domain sources properly estimated the location of epileptogenic zones (see Fig. 5(c)). ESI of the raw EEG and subsequent $S_{\mathrm{CFC}}$ mapping of the re- sulting sources also estimates an approximate EZ localization (see Fig. 5, Fig. 6). However, the resulting estimate is not as precise as that from the proposed method. This may be due to artifact skewing the ESI beyond the ability of the $\mathrm{S}_{\mathrm{CFC}}$ to recover. Furthermore, $\mathrm{S}_{\mathrm{CFC}}$ computation in the source domain is computationally more intensive than that from scalp EEG directly. Other techniques, such as that proposed by Jiang et al [55], could reduce the computational burden of estimating the LFR and HFR for $\mathrm{S}_{\mathrm{CFC}}$ construction in the source domain.

$\mathrm{S}_{\mathrm{CFC}}$ signal was derived from interacting HFR and LFR without artifact preprocessing, such as Fig. 2(e), simulation results showed that these interactions were selective for brain rhythms over ictal artifacts from muscle, and that the interacting activities of neural rhythms were stronger than other activities in the modulated frequency ranges (see Fig. 3). Phase selectivity of the amplitude of the high frequencies furthermore 


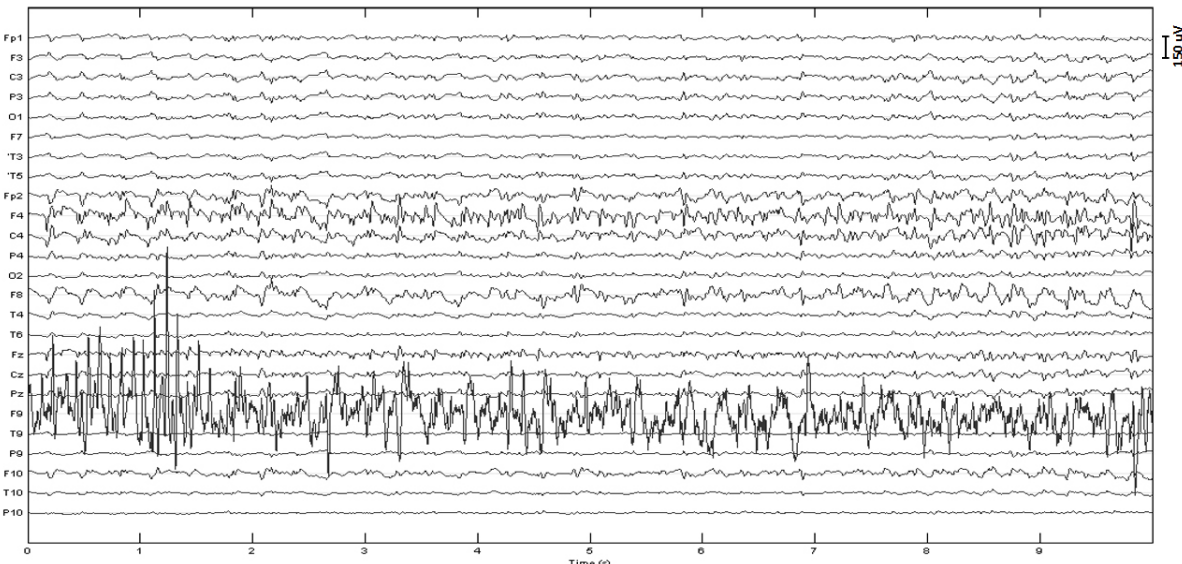

(a)

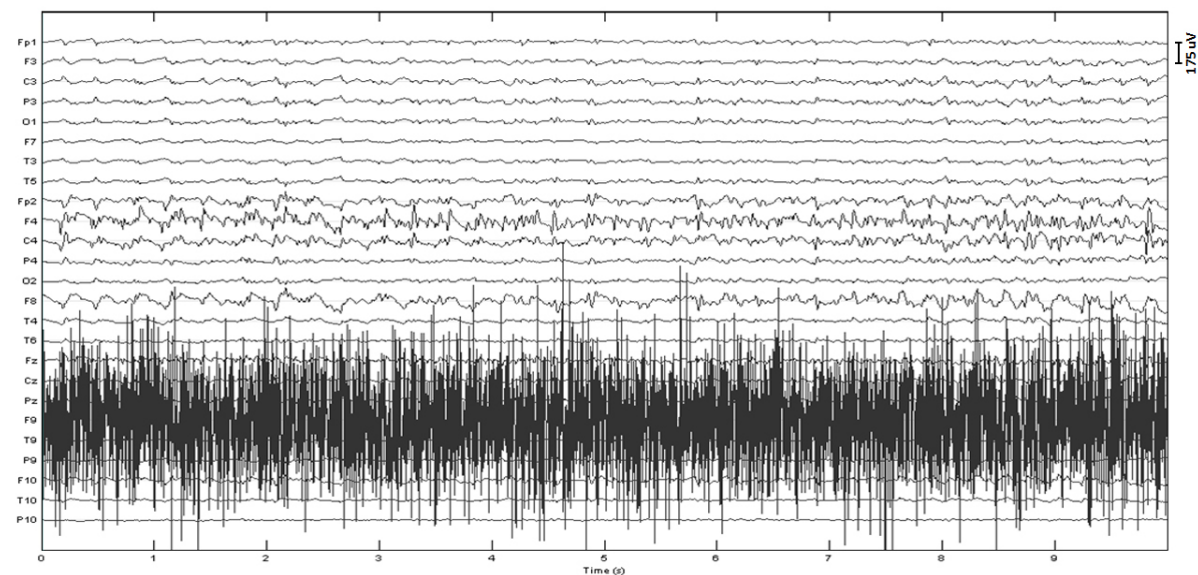

(c)

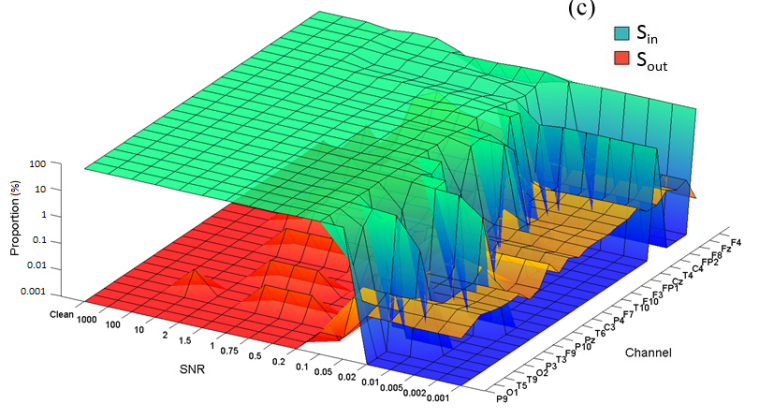

(e)

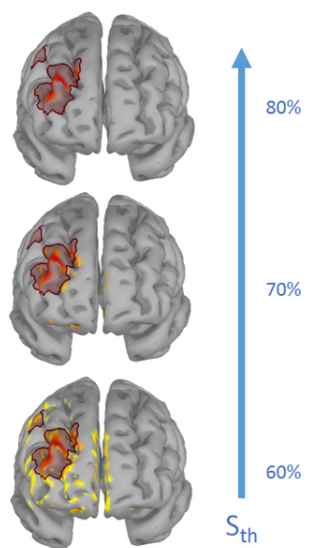

(b)

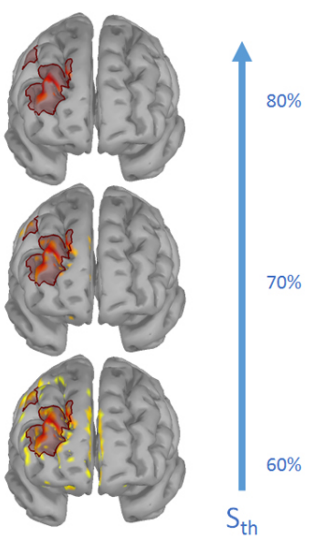

(d)

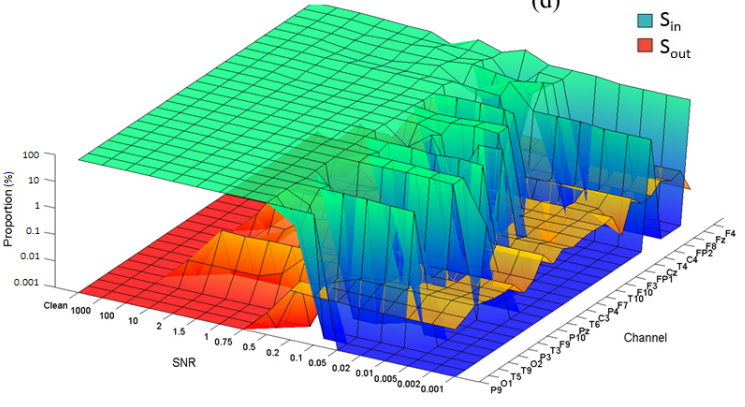

(f)

Fig. 8. Sensitivity and specificity of the $\mathrm{S}_{\mathrm{CFC}}$ localization technique in the presence of artifact added to individual electrodes. Examples of EEG traces from seizure one from patient P3 are shown with electrode F9 contaminated by (a) EMG at an SNR of 0.05, and (c) GWN at 0.1 SNR. The amplitude of the projected traces was scaled according to variance matching by the ratio of the standard deviation of the projection to that of the scalp EEG recording at the F4 electrode. In low SNR cases such as these, the $\mathrm{S}_{\mathrm{CFC}}$-mapped source estimates may lie outside the ROI using the $3 \mathrm{~dB}$-below-max threshold, $S_{t h}$. However, extraneous sources can be eliminated by increasing $S_{t h}$ as demonstrated in (b) and (d). Contamination simulations were performed across all electrodes for both noise types at varying SNR to produce an ROC-type analysis shown in (e) and (f). Electrodes are arranged by distance from the ROI. The $S_{i n}$ surface represents the percent of the ROI recovered (sensitivity), and the $S_{\text {out }}$ surface represents the percent of non-ROI identified (1-specificity).

seems to remove EMG artifacts. Comparing effects between EMG artifacts and white noise, the white noise affected the coupling more negatively than uncorrelated EMG signals (see Fig. 8(e) and (f)).

\section{B. $S_{C F C}$ signal is suitable to localize sources of seizure genesis}

ESI estimates corrupted by EMG artifact are unreliable because a solution space that only allows intra-cranial dipoles cannot account for a scalp-recording that arises from both intra and extra-cranial sources [56]. ESI estimates from $\mathrm{S}_{\mathrm{CFC}}$ demonstrated significant advantages over those from "raw" scalp EEG, because they are more robust to corruption by EMG (see Fig. 8(b)) or movement (see Fig. 4) artifacts. The method proposed in this paper requires only prior elimination of problematic traces such as those demonstrating loss of electrode contact rather than subjective artifact labeling. By using $\mathrm{S}_{\mathrm{CFC}}$ signal in ESI, modelling investigations indicated that an SNR greater than 0.2 can achieve successful localization regarding EMG artifacts (see Fig. 8). In comparison with scalp EEG measurements, illustrative worst-case estimates of 
TABLE II

SOURCE LOCALIZATION RESULTS

\begin{tabular}{|c|c|c|c|c|c|c|c|c|}
\hline \multirow[b]{2}{*}{ P\# } & \multirow[b]{2}{*}{$\mathrm{EC}$} & \multirow[b]{2}{*}{$\mathrm{SR}(\mathrm{Hz})$} & \multirow[b]{2}{*}{ Sz\# } & \multicolumn{2}{|c|}{ Ranges $(\mathrm{Hz})$} & \multicolumn{2}{|c|}{ Localized ROI } & \multirow{2}{*}{$\begin{array}{c}\text { Surgical } \\
\text { Resection }\end{array}$} \\
\hline & & & & LFO & $\mathrm{HFO}$ & $\mathrm{S}_{\mathrm{CFC}}$ & Raw & \\
\hline \multirow[t]{2}{*}{ P1 } & \multirow[t]{2}{*}{-} & \multirow[t]{2}{*}{1000} & 1 & $1.1-1.6$ & $70-110$ & Left: F & Right: PO & \multirow[t]{2}{*}{ Left: FT } \\
\hline & & & 2 & $1.1-1.8$ & $70-110$ & Left: F & Right: P & \\
\hline \multirow[t]{3}{*}{$\mathrm{P} 2$} & \multirow[t]{3}{*}{ I } & \multirow[t]{3}{*}{512} & 1 & $2.4-3.2$ & $35-50$ & Right: T & Right: O & \multirow[t]{3}{*}{ Right: $\mathrm{T}$} \\
\hline & & & 2 & $3.4-4.4$ & $32-40$ & Right: $\mathrm{T}$ & Left: $\mathrm{T}$ & \\
\hline & & & 3 & $1.1-1.3$ & $24-60$ & Right: T & Right: O & \\
\hline \multirow[t]{2}{*}{ P3 } & \multirow[t]{2}{*}{ I } & \multirow[t]{2}{*}{500} & 1 & $3.0-4.2$ & $20-50$ & Right: F & Left: $\mathrm{T}$ & \multirow[t]{2}{*}{ Right: F } \\
\hline & & & 2 & $3.2-4.3$ & $24-35$ & Right: F & Left: $\mathrm{T}$ & \\
\hline \multirow[t]{3}{*}{ P4 } & \multirow[t]{3}{*}{ I } & \multirow[t]{3}{*}{500} & 1 & $1.0-1.4$ & $30-70$ & Left: F & Right: F & \multirow[t]{3}{*}{ Left: F } \\
\hline & & & 2 & $1.0-1.5$ & $30-80$ & Left: F & Right: F & \\
\hline & & & 3 & $1.0-2.5$ & $45-65$ & Left: F & Right: F & \\
\hline \multirow[t]{2}{*}{ P5 } & \multirow[t]{2}{*}{ I } & \multirow[t]{2}{*}{256} & 1 & $2.6-2.9$ & $50-80$ & Left: $\mathrm{T}$ & Right: F, Left: $T$ & \multirow[t]{2}{*}{ Left: $\mathrm{T}$} \\
\hline & & & 2 & $1.0-1.5$ & $35-60$ & Left: $\mathrm{T}$ & Right: F, Left: T & \\
\hline \multirow[t]{3}{*}{ P6 } & \multirow[t]{3}{*}{ I } & \multirow[t]{3}{*}{250} & 1 & $2.9-3.9$ & $57-77$ & Left: F & Right: F & \multirow[t]{3}{*}{ Left: DF } \\
\hline & & & 2 & $1.5-2.4$ & $50-80$ & Left: T, Right: $\mathrm{T}$ & Right: F, Left: F & \\
\hline & & & 3 & $1.0-2.0$ & $50-80$ & Left: FT & Right: FP & \\
\hline \multirow[t]{2}{*}{ P7 } & \multirow[t]{2}{*}{ I } & \multirow[t]{2}{*}{200} & 1 & $1.0-2.0$ & $35-55$ & Right: $\mathrm{T}$ & Left: $\mathrm{O}$ & Right: $\mathrm{T}$ \\
\hline & & & 2 & $1.0-1.4$ & $40-70$ & Left: $\mathrm{T}$ & Left: $\mathrm{T}$, Right: $\mathrm{T}$ & \\
\hline P8 & II & 200 & 1 & $2.9-3.3$ & $58-65$ & Left: PO & Right: TF & Left: P \\
\hline P9 & III & 200 & 1 & $1.0-1.5$ & $50-60$ & Left: $\mathrm{O}$ & Right: F & Left: FT \\
\hline P10 & IV & 200 & 1 & $1.0-1.3$ & $70-80$ & Right: FT & Right: PO & Right: DF \\
\hline
\end{tabular}

F: frontal; T: temporal; P: parietal; O: occipital; D: dorsolateral; -: unknown; P\#: patient number; EC: Engel

Class (Engel 1993); SR: Sampling Rate; Sz\#:seizure number.

SNR at different frequency ranges from patient P3 were 2.0 (for 1-3 Hz), 1.2 (for 3-5 Hz), 0.89 (for 5-30 Hz), 0.55 (for $30-70 \mathrm{~Hz}$ ), and 0.51 (for 70-125 Hz) (see Fig. 7(b)). Our results show that the sROI were concordant with the resection in Engel Class I patients. When multiple seizures of the same patient were available, the consistency of localization between seizures could further validate sROI (see Table II).

\section{Effects of sampling frequency on scalp EEG recordings}

Ten seizures from patient P1-P4 (Engel Class I) with a sampling frequency $\geq 500 \mathrm{~Hz}$ produced a sROI concordant with each patient's resection. For sampling frequencies less than $500 \mathrm{~Hz}$ only four out of seven seizures from patient P5P7 (Engel Class I) produced a concordant sROI (see Table II). We suggest that those results could be improved by using high sampling frequency. Conventional clinical recording used a sampling frequency of $200 \mathrm{~Hz}$ because it was suitable for visual analysis of EEG, but this is not suitable for computeraided surgical evaluations. The recorded signal at $200 \mathrm{~Hz}$ may have aliasing effects.

\section{Localization of $S_{C F C}$ signal can guide the placement of} intracranial electrodes

Where the sROI was not covered by subdural electrodes, the surgical outcome was no better than Engel Class II (see Fig. 9(a)). For patient P10 (Engel Class IV), the sROI did not match the resected region. The frontal region of the sROI was partially covered by the grid electrodes, and the temporal area of the sROI was not covered. For patient P9 (Engel Class III), the sROI was at the left occipital lobe, out of grid coverage. For patient P8 (Engel class II), two sROI were estimated. One was close to the resected region, but the second was not covered by the grid electrodes. For all of these patients, the sROI not covered by subdural electrodes may have been part of the EZ, as reported by an investigation of 36 surgical failures with focal neocortical epilepsy, where it was found that, in $28 \%$ of the failures, an additional EZ was present distant from the resection [57].

For patients P2-P5 (Engel Class I) and patient P1, the sROI agreed with the resected region. For patients P6 and P7 (Engel Class I), there was agreement between the sROI and the resected region for one out of three and one out of two seizures, respectively. Perhaps this is due to the low sampling rate. The cross frequency interactions between LFR and HFR are associated with the EZ (see Table II). Delta rhythms 

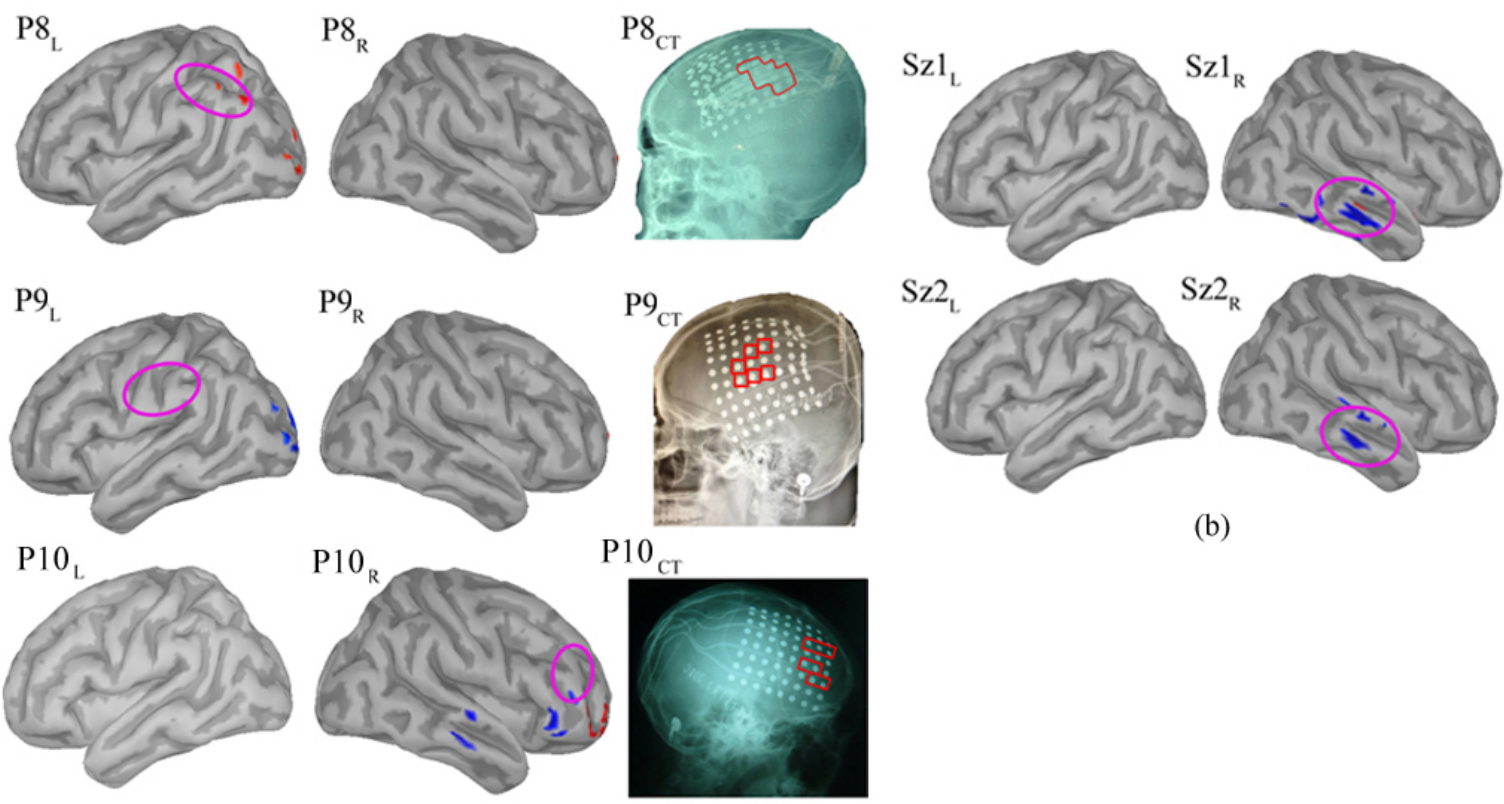

$\mathrm{P} 10$

(b)

(a)

Fig. 9. Source localization results and coupled frequency ranges. (a) Source activity of patient $\mathrm{P} 8, \mathrm{P} 9$, and $\mathrm{P} 10$. (P8 $8_{\mathrm{L} / \mathrm{R}}$ ) Left and right view of source activity of patient P8 seizure one. Patient P8 achieved Engel Class II outcome. $8_{\mathrm{CT}}$ was the CT imaging with intracranial grid of patient $\mathrm{P} 8$. (P9 $\left./ \mathrm{R}\right)$ Left and right view of source activity of patient P9 seizure one. Patient P9 achieved Engel Class III outcome. $\mathrm{P} 9{ }_{\mathrm{CT}}$ was the CT imaging with intracranial grid of patient P9. $\left(\mathrm{P} 10_{\mathrm{L} / \mathrm{R}}\right)$ Left and right view of source activity of patient P10 seizure one. Patient P10 achieved Engel Class IV outcome. P10 $\mathrm{CT}$ was the CT imaging with intracranial grid of patient P10. (b) Source activity of patient P2 with Engel Class I. Left and right view of localized source activity from the $\mathrm{S}_{\mathrm{CFC}}$ signal of patient $\mathrm{P} 2$ 's seizure one $\left(\mathrm{Sz}_{\mathrm{L} / \mathrm{R}}\right)$ and seizure two $\left(\mathrm{Sz}_{\mathrm{L} / \mathrm{R}}\right)$. The purple ellipse marks the resected area in all figures.

have been commonly reported in seizures [27]-[29], [31]. High gamma rhythms $(40-120 \mathrm{~Hz})$ increased in amplitude in human intracranial recordings at seizure onset [58]. Others have demonstrated that the surgical removal of regions generating increased ictal high frequency oscillations correlates positively with a seizure-free post-surgical outcome [25], [33], [36], [59]. Our group has previously reported low frequency oscillation modulated high frequency oscillation can be used to successfully define ROI using intracranial recordings [26]. More recently, cross frequency coupling between high (gamma and ripple) and low (delta, theta, alpha, and beta) frequency rhythms was reported to be significantly stronger in the SOZ compared to normal regions in intracranial recording of deep sleep [60].

\section{CONCLUSION}

Rhythmic features within the scalp EEG at electrographic seizure onset showed cross frequency coupling. The $\mathrm{S}_{\mathrm{CFC}}$ signal is proposed as a novel technique which extracts intracranial EEG features from noisy scalp recordings. Simulation validated that localization using the $\mathrm{S}_{\mathrm{CFC}}$ is robust in low SNR conditions. sROI agree with resected regions of patients with varying Engel Class scores, assuming high-frequency sampling rates greater than or equal to $500 \mathrm{~Hz}$. Therefore we propose that $\mathrm{S}_{\mathrm{CFC}}$-based ESI is a useful construct for estimating the EZ from scalp recordings. This approach could enhance the placement of intracranial electrode for surgical intervention of epilepsy.

\section{ACKNOWLEDGMENT}

The authors would like to thank Sinisa Colic for help regarding MATLAB codes.

\section{REFERENCES}

[1] P. Kwan and M. J. Brodie, "Early identification of refractory epilepsy," N. Engl. J. Med., vol. 342, pp. 314-319, 2000

[2] F. Rosenow and H. Lüders, "Presurgical evaluation of epilepsy," Brain, vol. 124, pp. 1683-1700, 2001.

[3] B. Diehl and H. O. Lüders, "Temporal lobe epilepsy: when are invasive recordings needed?" Epilepsia, vol. 41, pp. S61-74, 2000.

[4] J. Yuan et al., "Intracranial electrodes in the presurgical evaluation of epilepsy," Neurol. Sci., vol. 33, pp. 723-729, 2012.

[5] D. J. Englot et al., "Epileptogenic zone localization using magnetoencephalography predicts seizure freedom in epilepsy surgery," Epilepsia, vol. 56, pp. 949-958, 2015.

[6] C. M. Michel et al., "EEG source imaging," Clin Neurophysiol., vol. 115, pp. 2195-2222, 2004.

[7] B. He et al., "Electrophysiological imaging of brain activity and connectivity-challenges and opportunities," IEEE Trans. Biomed. Eng., vol. 58, no. 7, pp. 1918-1931, Jul. 2011.

[8] B. J. Edelman, et al., "EEG source imaging enhances the decoding of complex right-hand motor imagery tasks," IEEE Trans. Biomed. Eng., vol. 63, no. 1, pp. 4-14, Jan. 2016.

[9] V. Brodbeck et al., "Electroencephalographic source imaging a prospective study of 152 operated epileptic patients," Brain, vol. 134, pp. 28872897, 2011.

[10] K. Kaiboriboon et al., "EEG source imaging in epilepsy - practicalities and pitfalls," Nat. Rev. Neurol., vol. 8, pp. 498-507 2012.

[11] K. Elisevich et al., "An assessment of MEG coherence imaging in the study of temporal lobe epilepsy," Epilepsia, vol. 52, pp. 1110-1119, 2011.

[12] Y. Lu et al., "Seizure source imaging by means of FINE spatio-temporal dipole localization and directed transfer function in partial epilepsy patients," Clin. Neurophysiol., vol. 123, pp. 1275-1283, 2012.

[13] S. M. Bowyer, "Coherence a measure of the brain networks: past and present," Neuropsychiatric Electrophysiology, vol. 2, p. 1, 2016. 
[14] A. Coito et al., "Altered directed functional connectivity in temporal lobe epilepsy in the absence of interictal spikes: A high density EEG study," Epilepsia, vol. 57, pp. 402-411, 2016.

[15] S. L. Gonzalez Andino et al., "Non-stationary distributed source approximation: an alternative to improve localization procedures," Hum. Brain Mapp. vol. 14, pp. 81-95, 2001.

[16] K. Sekihara et al., "Time-frequency MEG-MUSIC algorithm," IEEE Trans. Med. Imaging., vol. 18, pp. 92-97, 1999.

[17] A. M. Lascano et al., "A review on non-invasive localisation of focal epileptic activity using EEG source imaging," Epileptologie, vol. 29, pp. 80-89, 2012.

[18] L. Ding et al., "Ictal source analysis: localization and imaging of causal interactions in humans," Neuroimage, vol. 34 pp. 575-586, 2007.

[19] L. Yang et al., "Dynamic imaging of ictal oscillations using non-invasive high-resolution EEG," Neuroimage, vol. 56, pp. 1908-1917, 2011.

[20] N. Mammone and F. Morabito, "Enhanced automatic artifact detection based on independent component analysis and Renyi's entropy," Neural Networks, vol. 21, pp. 1029-1040, 2008.

[21] J. S. Ebersole and S. V. Pacia, "Localization of temporal lobe foci by ictal EEG patterns," Epilepsia, vol. 37, pp. 386-399, 1996.

[22] G. Buzsáki, Rhythms of the brain. New York, NY, USA: Oxford Univ. Press, 2006, ch. 12, pp. 335-243.

[23] J. E. Lisman and M. A. P. Idiart, "Storage of $7 \pm 2$ short-term memories in oscillatory subcycle," Science, vol. 267, vol. 5203, pp. 1512-1515, 1995.

[24] A. K. Engel et al., "Dynamic predictions: oscillations and synchrony in top-down processing," Nat. Rev. Neurosci, vol. 2, pp. 704-716, 2001.

[25] M. Cotic et al., "Spatial coherence profiles of ictal high-frequency oscillations correspond to those of interictal low-frequency oscillations in the ECoG of epileptic patients," IEEE Trans. Biomed. Eng., vol. 63, no. 1, pp. 76-85, Jan. 2016.

[26] M. Guirgis et al., "Defining regions of interest using cross frequency coupling in extratemporal lobe epilepsy patients," J. Neural Eng., vol. 12, p. 026011 , Mar. 2015.

[27] D. Panet-Raymond and J. Gotman, "Asymmetry in delta activity in patients with focal epilepsy," Electroencephalogr. Clin. Neurophysiol., vol. 75, pp. 474-481, 1990.

[28] J. R. Hughes and J. J. Fino, "EEG in seizure prognosis: association of slow wave activity and other factors in patients with apparent misleading epileptiform findings," Clin. EEG Neurosci., vol. 35, pp. 181-184, 2004.

[29] F. Brigo, "Intermittent rhythmic delta activity patterns," Epilepsy Behav, vol. 20, pp. 254-256, 2011.

[30] A. Schnitzler and J. Gross, "Normal and pathological oscillatory communication in the brain," Nat. Rev. Neurosci, vol. 6, pp. 285-296, 2005.

[31] J. X. Tao et al., "Interictal regional delta slowing is an EEG marker of epileptic network in temporal lobe epilepsy," Epilepsia, vol. 52, pp. 467-476, 2011.

[32] J. A. Blanco et al., "Data mining neocortical high-frequency oscillations in epilepsy and controls," Brain, vol. 134, pp. 2948-2959, 2011.

[33] J. Jacobs et al., "High-frequency electroencephalographic oscillations correlate with outcome of epilepsy surgery," Ann. Neurology, vol. 67, pp. 209-220, 2010

[34] J. Jacobs et al., "High-frequency oscillations (HFOs) in clinical epilepsy," Prog. Neurobiology, vol. 98, pp. 302-315, 2012.

[35] Y. Lu et al., "Noninvasive imaging of the high frequency brain activity in focal epilepsy patients," IEEE Trans. Biomed. Eng., vol. 61, no. 6, pp. 1660-1667, Jun. 2014.

[36] J. Y. Wu et al., "Removing interictal fast ripples on electrocorticography linked with seizure freedom in children," Neurology, vol. 75, pp. 16861694,2010

[37] T. Akiyama et al., "Focal resection of fast ripples on extraoperative intracranial EEG improves seizure outcome in pediatric epilepsy," Epilepsia, vol. 52, pp. 1802-1811, 2011.

[38] K. Kobayashi et al., "Scalp-recorded high-frequency oscillations in childhood sleep-induced electrical status epilepticus," Epilepsia, vol. 51, pp. 2190-2194, 2010.

[39] T. Demiralp et al., "Gamma amplitudes are coupled to theta phase in human EEG during visual perception," Int. J. Psychophysiol., vol. 64, pp. 24-30, 2007.

[40] U. Friese et al., "Successful memory encoding is associated with increased cross-frequency coupling between frontal theta and posterior gamma oscillations in human scalp-recorded EEG," NeuroImage, vol. 66, pp. $642-647,2013$

[41] A. B. L. Tort et al., "Measuring phase-amplitude coupling between neuronal oscillations of different frequencies," J. Neurophysiol., vol. 104, pp. 1195-1210, 2010.
[42] D. Dvorak and A. A. Fenton, "Toward a proper estimation of phaseamplitude coupling in neural oscillations," J. Neurosci.Methods, vol. 225, pp. 42-56, 2014.

[43] M. Cotic et al., "Epilepsia mapping the coherence of ictal high frequency oscillations in human extratemporal lobe epilepsy," Epilepsia, vol. 56, pp. 393-402, 2015.

[44] A. Bruns, "Fourier-, Hilbert- and wavelet-based signal analysis: are they really different approaches?" J. Neurosci. Methods, vol. 137, pp. 321-332, 2004.

[45] F. Tadel et al., "Brainstorm: A user-friendly application for MEG/EEG analysis," Comput. Intell. Neurosci., vol. 2011, p. 879716, Jan. 2011.

[46] J. Z. Wang et al., "Magnetic source images determined by a lead-field analysis: the unique minimum-norm leastsquares estimation," IEEE Trans. Biomed. Eng., vol. 39, no. 7, pp. 665-675, Jul. 1992.

[47] R. D. Pascual-Marqui, "Standardized low-resolution brain electromagnetic tomography (sLORETA): technical details," Methods Find. Exp. Clin. Pharmacol., vol. 24, pp. 5-12, 2002.

[48] C. J. Holmes et al., "Enhancement of MR images using registration for signal averaging," J. Comput. Assist. Tomogr., vol. 22, pp. 324-333, 1998.

[49] A. Gramfort et al., "OpenMEEG: opensource software for quasistatic bioelectromagnetics," Biomed. Eng. Online, vol. 9, no. 1, p. 45, 2010.

[50] C. K. Kovach et al., "Manifestation of ocular-muscle EMG contamination in human intracranial recordings," NeuroImage, vol. 54, pp. 213-233, 2011.

[51] S. D. Muthukumaraswamy, "High-frequency brain activity and muscle artifacts in MEG/EEG: a review and recommendations," Front. Hum. Neurosci., vol. 7, p. 00138, 2013.

[52] S. Makeig et al., Independent component analysis of electroencephalographic data, in Advances in Neural Information Processing Systems, vol. 8, D. Touretzky, M. Mozer and M. Hasselmo, Eds. Cambridge, MA, USA: MIT Press, 1996, pp. 145-151.

[53] A. Delorme et al., "Enhanced detection of artifacts in EEG data using higher-order statistics and independent component analysis," Neuroimage, vol. 34, pp. 1443-1449, 2007.

[54] I. I. Goncharova et al., "EMG contamination of EEG: spectral and topographical characteristics," Clin. Neurophysiol., vol. 114, pp. 15801593, 2003.

[55] H. Jiang et al., "Predictability of depression severity based on posterior alpha oscillations," Clin. Neurophysiol., vol. 127, pp. 2108-2114, 2016.

[56] B. W. McMenamin et al., "Validation of ICA-based myogenic artifact correction for scalp and source-localized EEG," Neuroimage, vol. 49, no. 3, p. 2416, 2010.

[57] D. J. Englot et al., "Factors associated with failed focal neocortical epilepsy surgery," Neurosurgery, vol. 75, pp. 648-656, 2014.

[58] R. S. Fisher et al., "High-frequency EEG activity at the start of seizures," J Clin Neurophysiol, vol. 9, pp. 441-448, 1992.

[59] H. Fujiwara et al., "Resection of ictal high-frequency oscillations leads to favorable surgical outcome in pediatric epilepsy," Epilepsia, vol. 53, pp. 1607-1617, 2012.

[60] M. Amiri et al., "Phase-Amplitude Coupling Is Elevated in Deep Sleep and in the Onset Zone of Focal Epileptic Seizures," Front. Hum. Neurosci., vol. 10, p. 387, 2016. 